\title{
Radioimmunoassay of plasma ouabain in healthy and pregnant individuals
}

\author{
O Vakkuri ${ }^{1}$, S S Árnason ${ }^{2}$, A Pouta ${ }^{3}$, O Vuolteenaho ${ }^{1}$ and \\ J Leppäluoto ${ }^{1}$ \\ ${ }^{1}$ Department of Physiology and Biocenter Oulu, University of Oulu, Finland \\ ${ }^{2}$ Department of Physiology, University of Reykjavik, Iceland \\ ${ }^{3}$ Department of Obstetrics and Gynecology, University Hospital, Oulu, Finland \\ (Requests for offprints should be addressed to J Leppäluoto, Department of Physiology, University of Oulu, P O Box 5000, 90014 Oulu, Finland; \\ Email: juhani.leppaluoto@oulu.fi)
}

\begin{abstract}
Ouabain was recently isolated from human plasma, bovine hypothalamus and bovine adrenal in attempts to identify endogenous substances inhibiting the cell membrane sodium pump. A number of radioimmunoassays have been developed in order to study the clinical significance of ouabain. The results have been controversial with regard to the presence and chemical nature of plasma ouabainlike immunoreactivity. We have now measured ouabain in healthy and pregnant individuals using solid-phase extraction of plasma samples followed by a new radioimmunoassay with the extraordinary sensitivity of at least $2 \mathrm{fmol} /$ tube $(5 \mathrm{pmol} / \mathrm{l})$. Plasma extracts, a previously isolated human plasma ouabain-like compound and bovine hypothalamic inhibitory factor displaced the tracer in parallel and eluted identically with ouabain in highperformance liquid chromatography. Plasma ouabain immunoreactivity was found to be much lower than reported previously: $12.6 \pm 1.3 \mathrm{pmol} / 1$ in healthy men
\end{abstract}

(mean \pm s.E., $n=20$ ) and $9 \cdot 4 \pm 0.7 \mathrm{pmol} / 1$ in women $(n=14)$. In pregnant women $(n=28)$ plasma ouabain concentration was $16 \cdot 3 \pm 4 \cdot 0 \mathrm{pmol} / 1$ during the first trimester, $18.8 \pm 4.3 \mathrm{pmol} / 1$ during the second trimester and $24 \cdot 3 \pm 4 \cdot 0 \mathrm{pmol} / 1$ during the third trimester (all $P<0 \cdot 01$ compared with non-pregnant women). Plasma ouabain 3-5 days after the delivery was $13 \cdot 6 \pm 1 \cdot 1 \mathrm{pmol} / 1(n=10$, $P<0 \cdot 05-0 \cdot 01$ compared with second and third trimesters). The pregnancy-related changes in the plasma concentrations of ouabain resembled those of cortisol. Therefore cortisol was measured from the same plasma samples and a significant positive correlation was found $(r=0.512$, $P=0.006)$. The similar profiles of plasma ouabain and cortisol during pregnancy and their rapid decreases postpartum are consistent with the adrenal cortical origin of ouabain and also show that the secretions of these hormones are possibly under the control of same factors. Journal of Endocrinology (2000) 165, 669-677

\section{Introduction}

The ubiquitous cell membrane enzyme $\mathrm{Na}^{+} / \mathrm{K}^{+}$-ATPase, the sodium pump, regulates the transmembrane sodium and potassium gradients. Inhibitors of the sodium pump such as digitalis glycosides have long been used in the treatment of heart failure and endogenous sodium pump inhibitors have been sought for decades. An endogenous ouabain-related compound has been isolated from human plasma (Hamlyn et al. 1991), bovine hypothalamus (Tymiak et al. 1993, Zhao et al. 1995, Kawamura et al. 1999) and bovine adrenals (Schneider et al. 1998). The isolated compounds were identical to plant-derived ouabain in several reverse phase HPLCs, mass spectrometry and hydrogen-1 nuclear magnetic resonance and also inhibited the sodium pump and increased the contractile force of atrial muscle. For some time it was believed that the endogenous compound was an isomer of ouabain (Tymiak et al. 1993, Zhao et al. 1995), but the isomerism was recently found to be caused by the artificial complexation of ouabain to borate from glassware used in the isolation process (Kawamura et al. 1999).

Normal pregnancy involves marked changes in endocrine functions in addition to the blood volume and vascular smooth muscle tone. Considering the observed adrenocortical origin of ouabain (Hamlyn et al. 1991, Schneider et al. 1998), the association of plasma ouabain with cortisol is of great interest, as the activation of the adrenal cortex during pregnancy is well known. Circulating endogenous cardiac glycoside-like compounds also link the sodium pump to vascular changes. In pregnant women, the lymphocyte sodium pump density and activity (Ang et al. 1990) and the erythrocyte sodium pump rate and activity (MacPhail et al. 1992, Yoshimura et al. 1993) have been found to be greater than in non-pregnant individuals. In contrast, the amount of the $2 \alpha$ subunit of 
the sodium pump in the myometrium is smaller in pregnant than in non-pregnant women (Maxwell et al. 1998). There are also many studies showing that the circulating concentrations or activities of digitalis- or ouabain-like compounds as measured by receptor assays or by immunoassays are greater in pregnant than in nonpregnant individuals (Graves et al. 1984, Valdes et al. 1985, Poston et al. 1989, Seely et al. 1992, Paci et al. 1996). However, the circulating concentrations of these compounds have been remarkably high and no detailed analyses concerning the nature of the biological activity or immunoreactivity measured as ouabain have been performed.

In order to study the secretion profile of ouabain and to understand the changes in blood pressure that occur during pregnancy, we decided to measure plasma concentrations of ouabain and cortisol in pregnant women. For this purpose we set up a new sensitive radioimmunoassay for ouabain and calibrated it with the previously isolated ouabain preparations of human plasma (Hamlyn et al. 1991) and bovine hypothalamus (Tymiak et al. 1993). We observed that plasma ouabain concentrations in healthy individuals were much lower than reported previously and that plasma ouabain and cortisol concentration profiles change in parallel during pregnancy, possibly reflecting a common regulation of these hormones.

\section{Materials and Methods}

\section{Study participants}

Informed consent was obtained from all the participants in the study. Blood samples were obtained from 14 nonpregnant female (mean age 32.7 years) and 20 male laboratory workers ( 43.2 years), and from 28 pregnant women $(28.9$ years) from the first $(n=10)$, second $(n=8)$ and third $(n=10)$ trimesters (gestational weeks 1-13, 14-25 and 26-40 respectively). A blood sample was also taken from 10 women (25.5 years) at 3-5 days after delivery. All the blood samples were taken between $0900 \mathrm{~h}$ and $1200 \mathrm{~h}$, into polypropylene or polystyrene test tubes containing EDTA as an anticoagulant. Plasma was stored at $-20^{\circ} \mathrm{C}$. Umbilical cord plasma and amniotic fluid samples were collected from six mothers $(30 \cdot 2$ years) after the delivery or amniotomy. Blood pressure was recorded by a sphygmomanometer with the person sitting after a 10-min rest. The pregnant women had normal pregnancies and delivered healthy babies.

\section{Chemicals}

Ouabain (G-strophantin) octahydrate, ouabagenin, digoxin, digitoxin and related steroids (aldosterone, cortisol, hydrocortisone, progesterone, $\beta$-estradiol, testosterone), bovine thyroglobulin and sodium cyanoborohydride were obtained from Sigma Chemical Company (St Louis, MO, USA). Freund's incomplete and complete adjuvants were purchased from Difco Laboratories (Detroit, MI, USA), L-tyrosine and polyethylene glycol 6000 from Fluka AG (Buchs, Switzerland), radioiodinated $\left({ }^{125} \mathrm{I}\right)$ ouabain derivative $(70-80 \mathrm{MBq} / \mathrm{nmol}$ ) from Biotop Ltd (Oulu, Finland), Sephadex G-50F from Pharmacia (Uppsala, Sweden). All other chemicals were obtained from E Merck AG (Darmstadt, Germany).

\section{Reference compounds}

Lyophilized preparations of the ouabain-like compound from human plasma (Hamlyn et al. 1991), approximately $1 \mathrm{pmol}$, and from bovine hypothalamus (Tymiak et al. 1993), 171 pmol, were generously supplied by Dr J M Hamlyn and Dr G T Haupert Jr respectively.

\section{Radioimmunoassays}

Ouabain was coupled to bovine thyroglobulin using the periodination and sodium cyanoborohydride method according to Masugi et al. (1986). The ouabainthyroglobulin conjugate was purified by Sephadex G-50 gel filtration. The conjugate was emulsified in Freund's complete adjuvant and injected subcutaneously at multiple sites on the backs of five rabbits $(1 \mathrm{mg}$ immunogen/rabbit). Boosters $(0.5 \mathrm{mg} /$ rabbit in Freund's incomplete adjuvant) were given at monthly intervals. All five rabbits produced antisera against ouabain after the primary immunization. The antiserum ' 199 ' at a final titer of 1:750 000, the ouabain tracer and ouabain standards or samples in duplicate were dissolved in the radioimmunoassay buffer (PBS with $0 \cdot 1 \%$ gelatin and $\mathrm{NaN}_{3}$, $\mathrm{pH} 7 \cdot 4$ ) and each pipetted in $100 \mu \mathrm{l}$ aliquots into polystyrene test tubes. After an overnight incubation at $6{ }^{\circ} \mathrm{C}$ the bound and free fractions were separated by double antibody precipitation in the presence of $8 \%$ polyethylene glycol, and the precipitates were counted for radioactivity. The sensitivity of the ouabain radioimmunoassay calculated at a level of $5 \%$ displacement of the tracer with antiserum 199 was $2 \mathrm{fmol} /$ tube or $5 \mathrm{pmol} / \mathrm{l}$ (Fig. 1). The ouabain concentrations in normal human plasma extracts varied between 3 and $10 \mathrm{fmol} /$ tube .

The within and between assay coefficients of variation were $3.8 \%$ and $13.4 \%$ at $15 \mathrm{pmol} / 1$ and $3.7 \%$ and $9.4 \%$ at $42 \mathrm{pmol} / 1$ respectively $(n=10)$. All the samples from pregnant and postpartum women were assayed in the same assay. Of the compounds tested, ouabagenin showed the greatest cross-reactivity (52\%); digoxin and digitoxin cross-reacted slightly $(1.7 \%)$, and other less relative compounds (butaline 1\%, dihydro-ouabain $0.7 \%$ and digoxigenin $0 \cdot 5 \%)$. Naturally occurring steroids cross-reacted very little: cortisol $0 \cdot 0005 \%$, aldosterone $0 \cdot 0008 \%$, progesterone $0.00017 \%$, estrone $0.00027 \%$, $\beta$-estradiol $0.0006 \%$, dehydroepiandrosterone $0.0003 \%$, hydroxyandrostenone $0 \cdot 0001 \%$ and testosterone $0 \cdot 0005 \%$ (Table 1). 


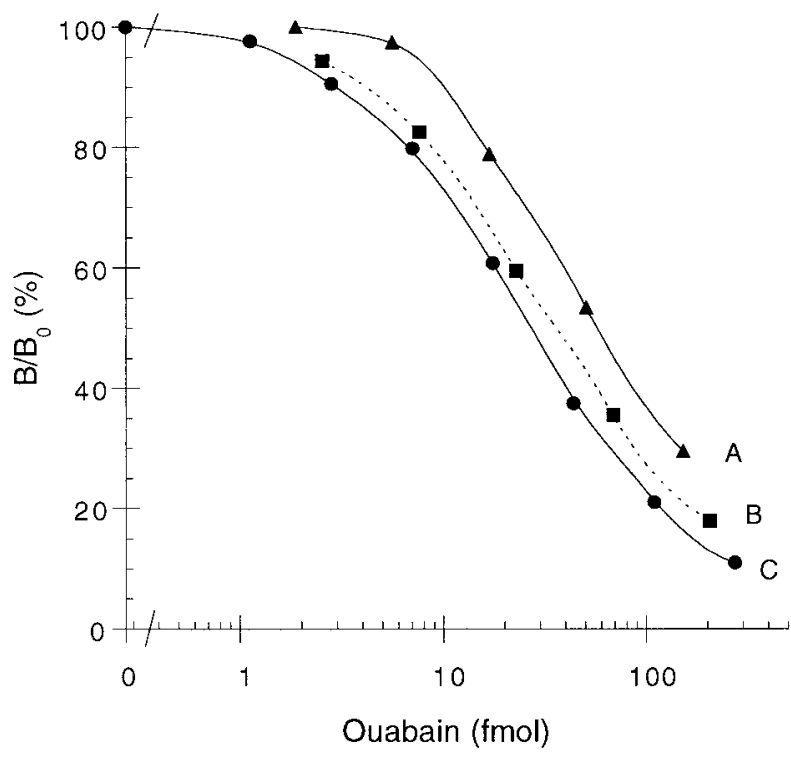

Figure 1 Displacement curves of the ouabain-like compound from human plasma ( $\boldsymbol{\Delta}, \mathrm{A}$; Hamlyn et al. 1991), the bovine hypothalamic inhibitory factor ( $\mathbf{\square}$, B; Tymiak et al. 1993) and commercial plant-derived ouabain $(\bullet, \mathrm{C})$ in the ouabain radioimmunoassay. Each point represents the mean of two measurements. $\mathrm{B} / \mathrm{B}_{\mathrm{O}}$, Percentage binding ratio over binding of the zero standard sample.

Plasma cortisol was measured using a Cortisol $\left({ }^{125} \mathrm{I}\right) \mathrm{kit}$ from Orion Diagnostica, Espoo, Finland. According to the manufacturer, the reference limits for samples taken between $0800 \mathrm{~h}$ and $1000 \mathrm{~h}$ are 189 and $682 \mathrm{nmol} / 1$ and the maximum intra-assay and interassay coefficients of variation are $4 \cdot 1$ and $9 \cdot 0 \%$, respectively. Cortisol was measured from the 27 plasma samples available to us at the time of the assay.

\section{Solid-phase separation of plasma and amniotic fluid samples}

Ouabain was extracted from 1-2 $\mathrm{ml}$ plasma or amniotic samples with Sep-Pak-Vac $500 \mathrm{mg}$ C18 cartridges (Waters, Milford, MA, USA) using an automated Gilson 5100 Aspec system and polypropylene test tubes. Briefly, the cartridge, preconditioned with $2 \mathrm{ml} 2$-propanol and $4 \mathrm{ml} 0 \cdot 1 \%$ aqueous trifluoroacetic acid (TFA), was loaded with the plasma sample to which $0 \cdot 2 \mathrm{ml} 1 \mathrm{M} \mathrm{HCl} / 1 \cdot 6 \%$ glycine/ml was added. The cartridge was washed with

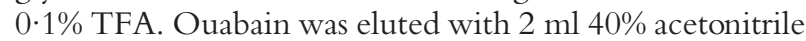
in $0.1 \%$ TFA and evaporated to dryness. The dried residue was reconstituted with $250 \mu \mathrm{l}$ radioimmunoassay buffer (see above). The recoveries of ouabain added to plasma were $103 \pm 2 \cdot 5 \%$ at $43 \mathrm{pmol} / 1$ and $94 \pm 1 \cdot 3 \%$ at $130 \mathrm{pmol} / 1$ (mean \pm S.E., $n=6)$.

\section{HPLC analysis of the reference materials and plasma extracts}

Aliquots of human plasma ouabain-like compound (Hamlyn et al. 1991) and bovine hypothalamic inhibitory factor (Tymiak et al. 1993) and Sep-Pak extracts of plasma or amniotic fluid (see above) were dissolved in $0 \cdot 1 \%$ TFA, centrifuged, filtered and subjected to reverse-phase HPLC using a Vydac C8 $218 \mathrm{TP}$ column $(0 \cdot 46 \times 15 \mathrm{~cm}$, Hesperia, CA, USA). A 30-min linear gradient from 5 to $30 \%$ acetonitrile in $0 \cdot 1 \%$ TFA was run at a flow rate of

Table 1 Comparison of immunoassays developed for ouabain with respect to specificity (cross-reactivity was determined at $50 \%$ displacement of tracer on a weight basis), sensitivity (read at 95\% binding level of tracer, or as mean for the zero calibrator - 2SD) and plasma ouabain (concentrations in healthy adults)

\begin{tabular}{|c|c|c|c|c|c|c|c|c|}
\hline & & Specificity $(\mathrm{C}$ & ss-reactivit & & & & & \\
\hline & Assay method & Ouabagenin & $\begin{array}{l}\text { Dihydro- } \\
\text { ouabain }\end{array}$ & Digoxin & Digitoxin & Steroids* & $\begin{array}{l}\text { Sensitivity } \\
(\mathrm{pmol} / \mathrm{l})\end{array}$ & $\begin{array}{l}\text { ouabain } \\
(\mathrm{pmol} / \mathrm{l})\end{array}$ \\
\hline Authors & & & & & & & & \\
\hline Harris et al. (1991) & ELISA & 40 & $0 \cdot 16$ & $5 \cdot 2$ & 28 & $\leq 0 \cdot 01$ & 100 & $100-180$ \\
\hline Doris et al. (1994) & ${ }^{3} \mathrm{H}-\mathrm{RIA}$ & $56 \cdot 2$ & - & $4 \cdot 5$ & - & $\leq 0.025$ & 80 & $<80$ \\
\hline Gomez-Sanchez et al. (1994) & ELISA & $2 \cdot 2$ & $0 \cdot 02$ & $<0 \cdot 001$ & $0 \cdot 12$ & $\leq 0.001$ & 30 & $30-50$ \\
\hline Lewis et al. (1994) & ELISA & $77 \cdot 2$ & - & $1 \cdot 25$ & - & $\leq 0.07$ & 60 & $<60$ \\
\hline Naruse et al. (1994) & ${ }^{3} \mathrm{H}-\mathrm{RIA}$ & - & - & $9 \cdot 2$ & - & $<0.01$ & 20 & $20-60$ \\
\hline Worgall et al. (1996) & ${ }^{3} \mathrm{H}-\mathrm{RIA}$ & 88 & - & 0.06 & $<0 \cdot 1$ & $<0 \cdot 1$ & 20 & $30-100$ \\
\hline Balzan et al. (1997) & ${ }^{3} \mathrm{H}-\mathrm{RIA}$ & - & - & $3 \cdot 8$ & 36 & $\leq 0 \cdot 18$ & 10 & $15-30$ \\
\hline Ferrandi et al. (1997) & ${ }^{3} \mathrm{H}-\mathrm{RIA}$ & 21 & - & $0 \cdot 4$ & 9 & $\leq 0 \cdot 9$ & 20 & $50-750$ \\
\hline Harwood et al. (1997) & EIA & 55 & $0 \cdot 3$ & $5 \cdot 3$ & 78 & $\leq 0 \cdot 02$ & 40 & $60-170$ \\
\hline Komiyama et al. (1997) & ELISA & 72 & $0 \cdot 82$ & $6 \cdot 4$ & $84 \cdot 6$ & $<0.01$ & 10 & $20-140$ \\
\hline Bernini et al. (1998) & ELISA $^{* *}$ & - & - & 3 & 5 & - & 10 & $10-175$ \\
\hline Butt et al. (1998) & ${ }^{3} \mathrm{H}-\mathrm{RIA}$ & $4 \cdot 0$ & $2 \cdot 0$ & $0 \cdot 4$ & 0.67 & $\leq 0.016$ & 60 & $400-800$ \\
\hline Present study & ${ }^{125}$ I-RIA & $52 \cdot 2$ & $0 \cdot 7$ & $1 \cdot 7$ & $0 \cdot 6$ & $<0.001$ & 5 & $10-20$ \\
\hline
\end{tabular}

*Aldosterone, cortisol, progesterone, $\beta$-estradiol, testosterone and other common steroids.

**Du Pont-New England Nuclear ELISA kit.

RIA, radioimmunoassay; ELISA, enzyme-linked immunosorbent assay; EIA, enzyme immunoassay. 
$1 \mathrm{ml} / \mathrm{min}$. Fractions of $1 \mathrm{ml}$ were collected into polypropylene test tubes, dried in Speed Vac, reconstituted with 250-500 $\mu \mathrm{l}$ radioimmunoassay buffer as described before and subjected to the ouabain radioimmunoassay. In the reverse-phase HPLC, $90 \%$ of the ouabain-like compound (Hamlyn et al. 1991) and 78\% of the bovine hypothalamic inhibitor (Tymiak et al. 1993) could be recovered.

\section{Statistical analyses}

The data are expressed as mean \pm s.E. For comparison of the ouabain or cortisol concentrations and blood pressure recordings between various experimental groups, one-way ANOVA followed by Newman-Keul's multiple comparison test was used. Spearman coefficients of rank correlation and linear correlation were calculated with immunoreactive plasma ouabain concentration as independent and systolic and diastolic blood pressure recordings or plasma cortisol as dependent variables.

\section{Results}

Validation of the radioimmunoassay for use with human plasma samples

The displacement curves of the bovine hypothalamic inhibitory factor and human plasma ouabain-like substance were parallel with that of plant-derived ouabain (Fig. 1). The apparent cross-reactivity of the hypothalamic inhibitory factor obtained from Dr Haupert (Tymiak et al. 1993) was $70 \%$ and that of plasma ouabain-like compound obtained from Dr Hamlyn (Hamlyn et al. 1991) was 52\%. Endogenous immunoreactivity present in human male plasma Sep-Pak extracts displaced the tracer in parallel with the ouabain standard (Fig. 2). The Sep-Pak extracts of the reference preparations from bovine and human plasma origin eluted identically with plant ouabain in the reverse phase HPLC system (Fig. 3). The recovery of the added or endogenous materials in the HPLC run was 78-103\%. When the ouabain-like compound isolated from human plasma was subjected to HPLC, a very small immunoreactive peak was found to elute after ouabain (Fig. 3B). Immunoreactive ouabain in Sep-Pak extracts of human pregnant plasma, umbilical cord plasma and amniotic fluid eluted in the reverse-phase HPLC system identically with plant ouabain (Fig. 4). These findings show that our radioimmunoassay is suitable for the measurement of endogenous ouabain.

\section{Plasma immunoreactive ouabain in reference and pregnant individuals}

The mean plasma ouabain concentrations measured by our radioimmunossay were $12.6 \pm 1.3 \mathrm{pmol} / 1$ in healthy men and $9 \cdot 4 \pm 0.7 \mathrm{pmol} / 1$ in women. The mean plasma

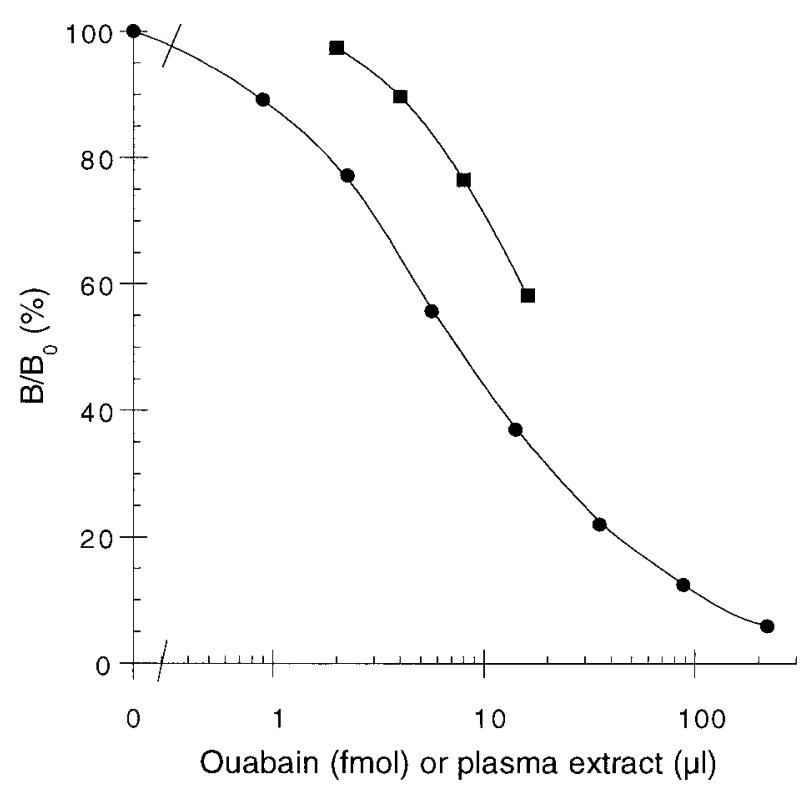

Figure 2 Displacement curves of ouabain $(-)$ and a human plasma extract ( $\mathbf{\square})$ in the ouabain radioimmunoassay. Each point represents the mean of two measurements. $B / B_{0}$, Percentage binding ratio over binding of the zero standard sample.

ouabain concentrations in healthy pregnant women during the first, second and third trimesters were $16 \cdot 3 \pm 4 \cdot 0$, $18 \cdot 8 \pm 4 \cdot 3$ and $24 \cdot 3 \pm 4 \cdot 0 \mathrm{pmol} / 1$ respectively; all these values are significantly greater than those in non-pregnant women $(P<0.01$; Fig. 5). Plasma ouabain concentration during the second and third trimesters was also significantly greater than that during the first trimester $(P<0 \cdot 05-$ $0 \cdot 01)$. After the delivery, plasma ouabain concentrations were $13 \cdot 6 \pm 1 \cdot 1 \mathrm{pmol} / 1$, significantly $(P<0 \cdot 05)$ lower than those during the second and third trimesters. Amniotic fluid and umbilical cord plasma ouabain levels were $25 \cdot 5 \pm 4 \cdot 1$ and $22 \cdot 0 \pm 2 \cdot 0 \mathrm{pmol} / 1$ respectively.

The mean systolic and diastolic blood pressures in healthy women were $127 \pm 4$ and $82 \pm 1 \mathrm{mmHg}$ respectively. In pregnant women the systolic blood pressure during the third trimester was significantly $(P<0 \cdot 05)$ greater than that during the first or second trimesters $(124 \pm 3 \mathrm{mmHg}$ compared with $110 \pm 3$ and $118 \pm 5 \mathrm{mmHg}$ respectively). The mean systolic and diastolic blood pressures in postpartum women were $125 \pm 3 \mathrm{mmHg}$ and $69 \pm 5 \mathrm{mmHg}$. There was a significant rank correlation between plasma ouabain concentration and the systolic blood pressure in pregnant women $(r=0.434, P=0.0269, n=26$; Fig. 6). The correlation between plasma ouabain and diastolic blood pressure in the same individuals was not significant $(r=0 \cdot 08, P>0 \cdot 05)$.

The mean morning plasma cortisol concentrations were $183 \pm 17 \mathrm{nmol} / 1$ during the first trimester, $214 \pm 44 \mathrm{nmol} / 1$ during the second trimester, $278 \pm$ $25 \mathrm{nmol} / 1$ during the third trimester and $146 \pm 12 \mathrm{nmol} / 1$ 


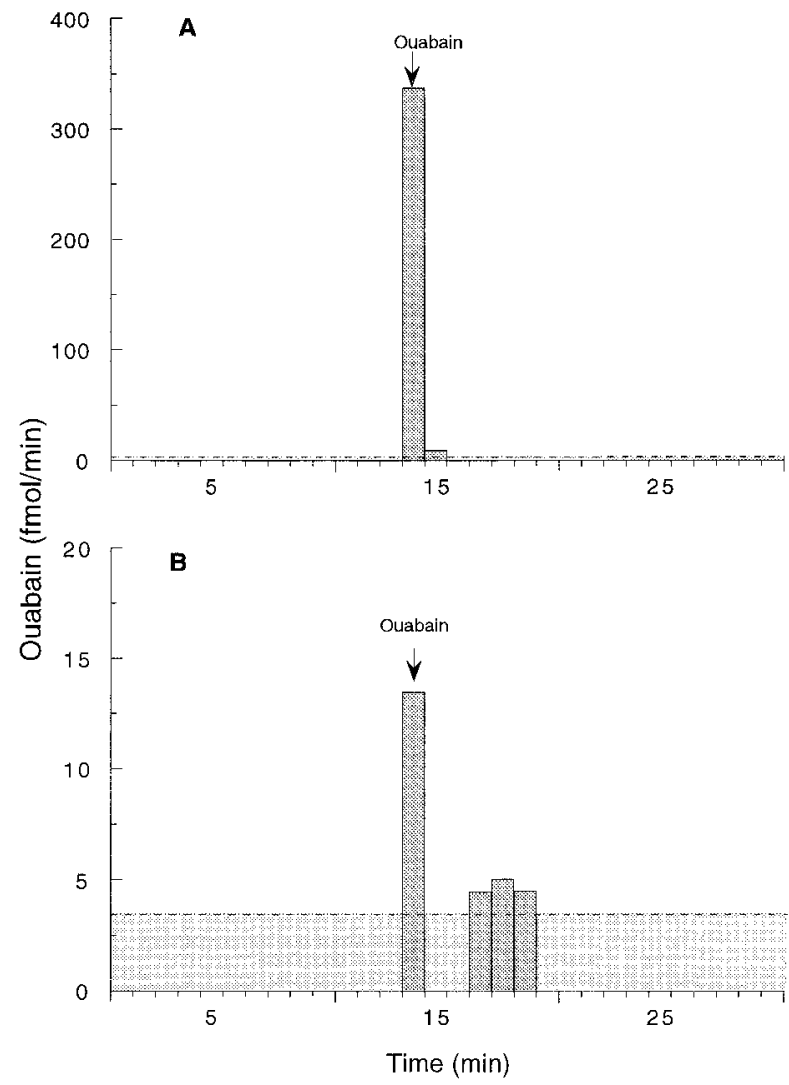

Figure 3 Reverse-phase HPLC of the bovine hypothalamic inhibitory factor (A) (Tymiak et al. 1993) and the ouabain-like compound from human plasma (B) (Hamlyn et al. 1991). The elution of plant-derived commercial ouabain is marked by an arrow. For HPLC conditions see methods. Dotted line represents the detection limit in the ouabain radioimmunoassay.

at 3-5 days after the delivery. The cortisol concentrations during the third trimester were significantly greater $(P<0 \cdot 05)$ than those during the first trimester or postpartum. There was a significant linear correlation between plasma ouabain and plasma cortisol concentrations in pregnant and postpartum women $(r=0.512, P=0.006$, $n=27$; Fig. 7). It should be stressed that our ouabain antiserum does not have any detectable cross-reaction with cortisol or the naturally occurring steroid hormones.

\section{Discussion}

In order to validate our ouabain radioimmunoassay for the analysis of human plasma samples, we compared the displacement curves of the human plasma endogenous ouabain-like compound (Hamlyn et al. 1991) and the bovine hypothalamic inhibitor (Tymiak et al. 1993) with commercial plant-derived ouabain. Both endogenous sub-
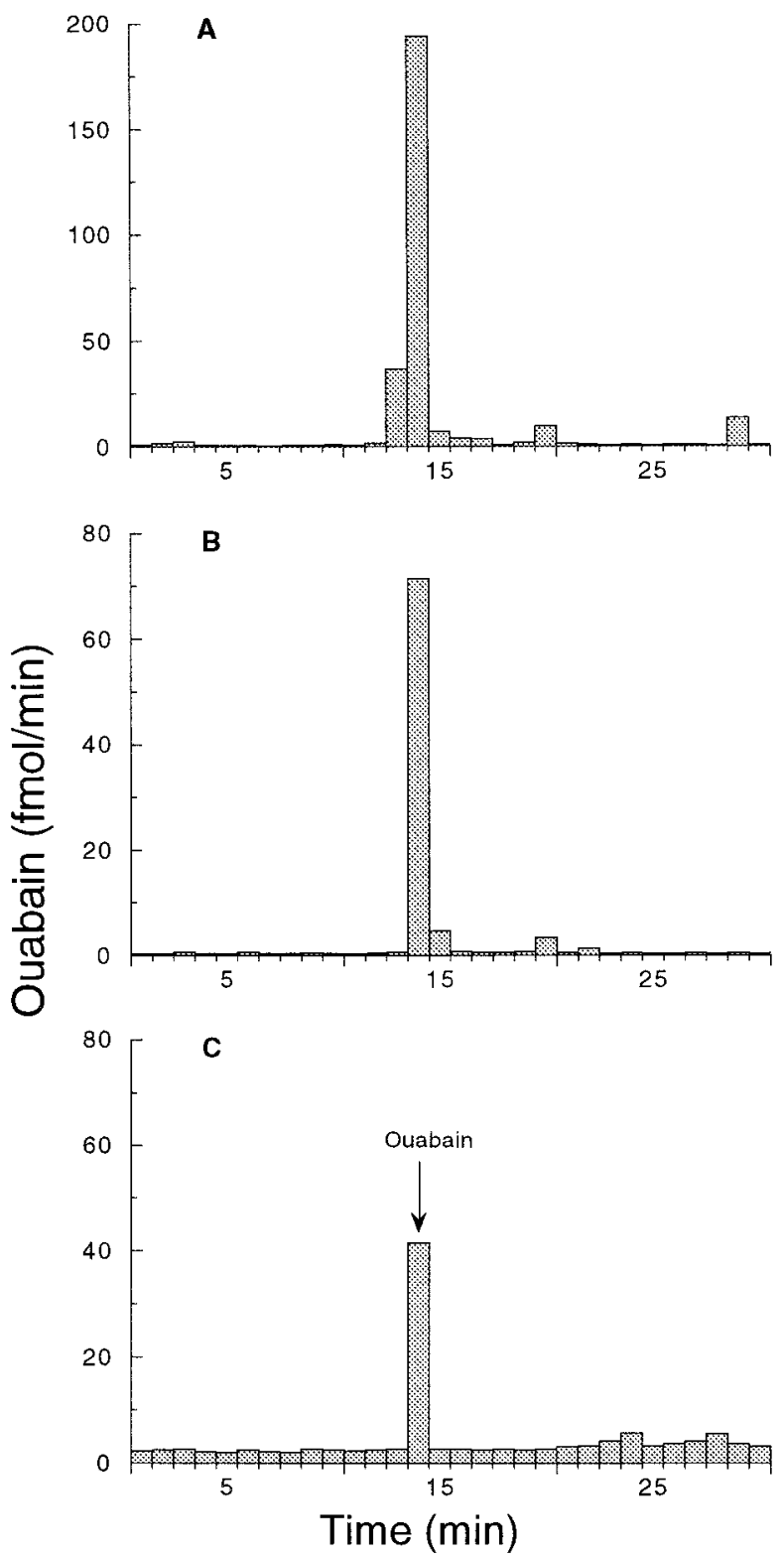

Figure 4 Reverse-phase HPLC of Sep-Pak extracts of plasma from a pregnant woman in the third trimester $(\mathrm{A})$, an amniotic fluid sample (B) and cord plasma (C). The elution of plant-derived ouabain is marked by an arrow in (C).

stances diluted in parallel with ouabain and their potencies compared with commercial plant ouabain were 52\% and $70 \%$ respectively. Considering the inaccuracies inherent in weighing small amounts of natural materials $(1 \mathrm{pmol}$ and $171 \mathrm{pmol})$, both endogenous substances probably are equipotent with ouabain in our radioimmunoassay. In addition, we wanted to see if the endogenous materials were homogenous. Reverse-phase HPLCs of both endogenous substances were performed and demonstrated almost 


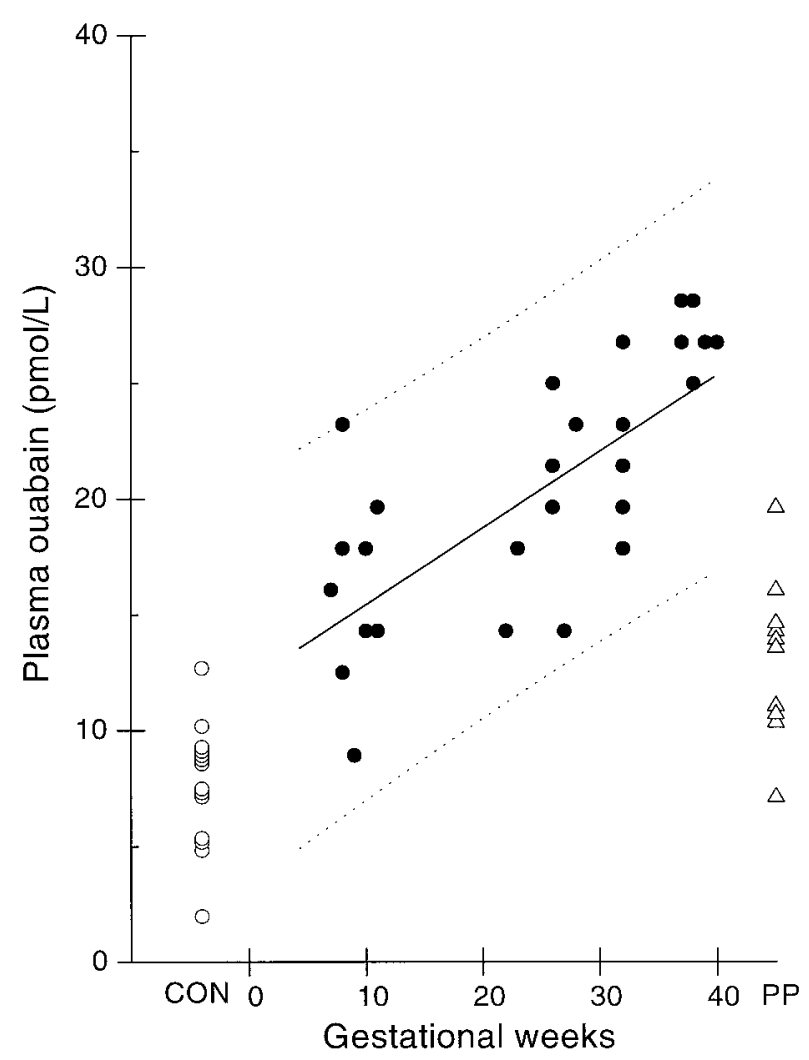

Figure 5 Plasma ouabain concentrations in non-pregnant women $(\mathrm{CON}, \bigcirc, n=14)$, and in pregnant women during gestational weeks $8-40(\bullet, n=28)$ and $3-5$ days after the delivery (PP, $\triangle$, $n=10$ ). There was a significant linear correlation between plasma ouabain and the duration of gestation (solid line). Predicition lines with $95 \%$ probability are shown (dotted lines).

complete recovery; in addition, the great majority (78$90 \%)$ of the immunoreactivity eluted identically with ouabain. However, a small broad immunoreactive peak eluted after ouabain in the human plasma preparation. This finding and the slightly lower than expected immunoreactivity of the human plasma and bovine hypothalamic preparations that we observed in this study may have been presumed to reflect the complexation of ouabain with borate from glassware that was recently shown to be possible (Kawamura et al. 1999); however, we did not use glassware in this study. Our radioimmunoassay is thus valid for the measurements of endogenous ouabain in biological fluids.

In the present study, the mean plasma ouabain concentrations in non-pregnant women were found to be $9.4 \mathrm{pmol} / 1$ and those in men were $12.4 \mathrm{pmol} / 1$. Previously, an enzyme-linked immunosorbent-assay (ELISA) method gave plasma ouabain concentrations of $138 \mathrm{pmol} / 1$ (Hamlyn et al. 1991). In other studies even greater concentrations have been reported, but the identity of the immunoreactivity was not studied (Masugi et al. 1986,

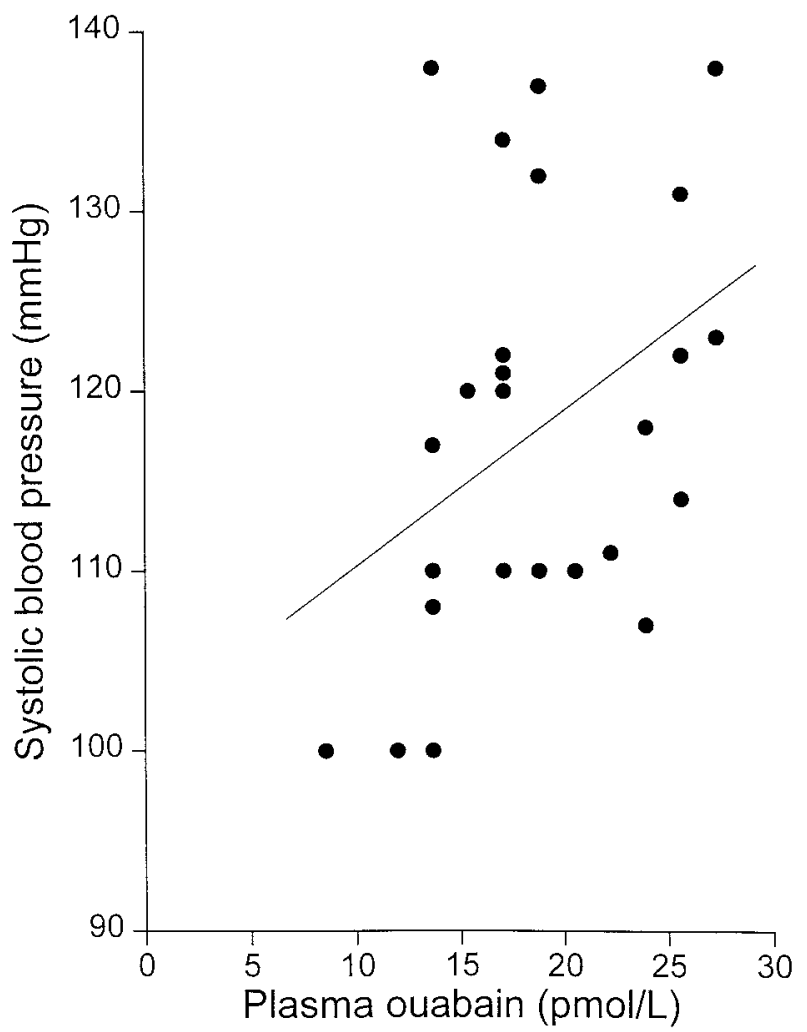

Figure 6 Systolic blood pressure as a function of plasma ouabain concentration in pregnant women. There was a significant positive rank correlation between the parameters (solid line: $r=0.434$, $P=0 \cdot 0269, n=26)$.

Harris et al. 1991, Gottlieb et al. 1992, Rossi et al. 1995). In a more recent radioimmunoassay study, plasma ouabain concentrations in healthy volunteers (sex not stated) were found to be $25 \mathrm{pmol} / 1$ and the immunoreactivity eluted in HPLC in the same way as ouabain (Balzan et al. 1997). In contrast, plasma ouabain concentrations less than $5 \mathrm{pmol} / 1$ have also been reported (Gomez-Sanchez et al. 1994, Doris et al. 1994, Lewis et al. 1994). A summary of the results of previous ouabain immunoassays was presented in Table 1. It is our opinion that the use of tritiated or enzyme-labeled tracers and the resulting low sensitivity of the previously used ouabain antisera are major reasons why greatly variable ouabain concentrations in human plasma have been reported. In addition, no attempts to compare the chemical and immunological properties of the endogenous ouabain-like substances with commercial ouabain have been made in any of the previous studies.

There are no previous studies reporting plasma immunoreactive ouabain concentrations in pregnant women. Using a radioreceptor assay, plasma ouabain concentrations in non-pregnant individuals were found to be $204 \mathrm{pmol} / 1$; those in pregnant women were up to $2000 \mathrm{pmol} / 1$ (Paci 


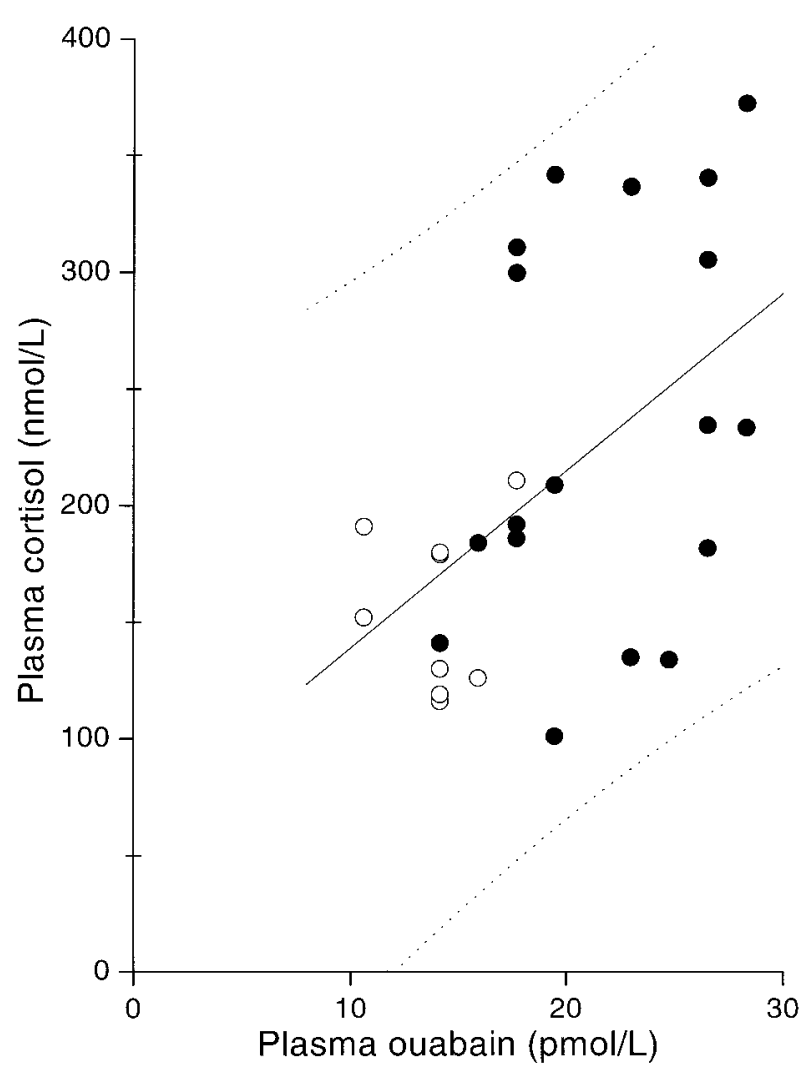

Figure 7 Plasma cortisol as a function of plasma ouabain in pregnant $(\bullet)$ and postpartum $(\bigcirc)$ women. There was a significant positive linear correlation between the parameters (solid line: $r=0.512, P=0.006, n=27)$. Prediction lines with $95 \%$ probability are shown (dotted lines).

et al. 1996). In contrast, plasma digitalis-like immunoreactivities have been measured in several studies. The concentrations in pregnant women have varied from 10 to $930 \mathrm{pmol} / \mathrm{l}$, but in none of these studies were analyses of the chemical identity of the measured immunoreactivity performed (Graves et al. 1984, Valdes \& Graves 1985, Poston et al. 1989, Seely et al. 1992, Paci et al. 1996). We have recently measured immunoreactive digitalis-like substances in human pregnant plasma and placenta after solid-phase extraction and HPLC and observed that the amount of immunoreactive digitalislike substance is much smaller than that of immunoreactive ouabain-like substance (S S Árnason, unpublished observation).

Systolic but not diastolic blood pressure in our pregnant women was significantly greater in the third trimester than in the first or second trimesters. We wanted therefore to know whether plasma ouabain concentrations correlated to blood pressure during pregnancy, and we observed a significant rank correlation between plasma ouabain concentration and systolic blood pressure but not between diastolic blood pressure. We also performed a linear correlation analysis between plasma ouabain and systolic blood pressure, but this did not reach statistical significance, indicating that the correlation is complex. It has been proposed that plasma ouabain could interfere with the sodium pump of vascular smooth muscle, resulting in increased vascular tone and increased blood pressure (Hamlyn \& Manunta 1992, Blaustein 1996). Our findings are consistent with this concept during pregnancy but not after the delivery, when plasma ouabain decreased but systolic blood pressure remained at increased values. The significant correlation between plasma ouabain and systolic blood pressure during pregnancy may still impart some role for ouabain in regulating blood pressure, but other factors have also to be taken into account.

In view of the observed adrenal origin of ouabain (Hamlyn et al. 1991, Schneider et al. 1998) and the finding that the stimulation of adrenal cells by adrenocorticotropic hormone $(\mathrm{ACTH})$ in vitro increases release of ouabain to the medium (Laredo et al. 1994), it was interesting to note that there was a gradual increase in maternal plasma ouabain during pregnancy and a rapid decrease within 3-5 days after the delivery in the present study. These changes resemble those in maternal plasma total cortisol concentration (Brien \& Dalrymple 1976, Cousins et al. 1983, Allolio et al. 1990). In our study, the mean plasma total cortisol concentration was $48 \%$ less in the postpartum women than in the third trimester women; in a similar study, a 25\% decrease was observed (Allolio et al. 1990). The difference is evidently due to the fact that our postpartum women were different from the third trimester ones.

We detected in this study high concentrations of ouabain in the amniotic fluid and cord plasma, indicating an association of ouabain with the fetoplacental unit. The significant positive linear correlation between the concentrations of ouabain and total cortisol in samples taken during pregnancy and postpartum further strengthens the proposal that ouabain and cortisol are under similar regulation in these conditions. The fluctuations in maternal cortisol have been accounted for by stimulation of adrenal cortex by ACTH and corticotropin-releasing hormone (Sasaki et al. 1989), an increase in cortisol-binding globulin, or an antiglucocorticoid action of progesterone (Brien \& Dalrymple 1976, Cousins et al. 1983, Allolio et al. 1990). The potential roles of these factors in determining plasma ouabain concentrations are not known.

In conclusion, we have developed a sensitive radioimmunoassay for ouabain that detects equally well plant-derived ouabain and the endogenous ouabain-like substance previously isolated from human plasma and bovine hypothalamus. Using the assay, we demonstrated that plasma ouabain concentrations in healthy individuals are much lower than reported previously and that plasma ouabain in pregnant women increases towards the delivery, abruptly decreases after it and correlates with plasma total cortisol, suggesting that the secretions of ouabain and 
cortisol during pregnancy may be regulated by the same factors.

\section{Acknowledgements}

We thank Ms Tuula Lumijärvi for expert technical assistance. This study was supported by grants from the Academy of Finland and the Icelandic University Fund to Dr Sighvatur S Árnason.

\section{References}

Allolio B, Hoffman J, Linton EA, Winkelmann W, Kusche M \& Schulte HM 1990 Diurnal salivary cortisol patterns during pregnancy and after delivery: relationship to plasma corticotrophin-releasing hormone. Clinical Endocrinology 33 279-289.

Ang LM, Taylor EA \& Oh VM 1990 Sodium pump numbers and cation transport of lymphocytes in pregnancy-induced hypertension. Journal of Hypertension 8 851-857.

Balzan S, Montali U, Di Bartolo V \& Ghione S 1997 Further evidence for an endogenous digitalis-like compound in newborn and adult plasma detected by anti-ouabain antiserum. Life Sciences 60 893-898.

Bernini G, Paci A, Sgro M, Moretti A \& Salvetti A 1998 Endogenous digitalis-like factors and ouabain immunoreactivity in adrenalectomized patients and normal subjects after acute and prolonged salt loading. American Journal of Hypertension 11 1-7.

Blaustein MP 1996 Endogenous ouabain: role in the pathogenesis of hypertension. Kidney International 49 1748-1753.

Brien TG \& Dalrymple IJ 1976 A longitudinal study of the free cortisol index in pregnancy. British Journal of Obstetrics and Gynaecology 83 361-367.

Butt AN, Semra YK, Lane SJ, Lee T \& Swaminathan R 1998 Endogenous ouabain secretion in man is not regulated by ACTH. Journal of Steroid Biochemistry and Molecular Biology 66 151-157.

Cousins L, Rigg L, Hollingsworth D, Meis P, Halberg F, Brink G \& Yen SSC 1983 Qualitative and quantitative assessment of the circadian rhythm of cortisol in pregnancy. American Journal of Obstetrics and Gynecology 145 411-416.

Doris PA, Jenkins LA \& Stocco DM 1994 Is ouabain an authentic endogenous mammalian substance derived from the adrenal? Hypertension 23 632-638. Obstetrics and Gynecology 145 411-416.

Ferrandi M, Manunta P, Balzan S, Hamlyn JM, Bianchi G \& Ferrari P 1997 Oubain-like factor quantification in mammalian tissues and plasma. Hypertension 30 886-896.

Gomez-Sanchez EP, Foecking MF, Sellers D, Blankenship MS \& Gomez-Sanchez CE 1994 Is the circulating ouabain-like compound ouabain? American Journal of Hypertension 7 647-650.

Gottlieb SS, Rogowski AC, Weinberg M, Krichten CM Hamilton BP \& Hamlyn JM 1992 Elevated concentrations of endogenous ouabain in patients with congestive heart failure. Circulation 86 420-425.

Graves SW, Valdes R Jr, Brown BA, Knight AB \& Craig HR 1984 Endogenous digoxin-immunoreactive substance in human pregnancies. Journal of Clinical Endocrinoogy and Metabolism $\mathbf{5 8}$ $748-751$.

Hamlyn JM, Blaustein MP, Bova S, DuCharme DW, Harris DW, Mandel F, Mathews WR \& Ludens JH 1991 Identification and characterization of a ouabain-like compound from human plasma. PNAS 88 6259-6263.

Hamlyn JM \& Manunta P 1992 Ouabain, digitalis-like factors and hypertension. Journal of Hypertension 10 (Suppl 7) S99-S111.

Harris DW, Clark MA, Fisher JD, Hamlyn JM, Kolbasa KP, Ludens JH \& DuCharme DW 1991 Development of an immunoassay for endogenous digitalislike factor. Hypertension 17 936-943.
Harwood S, Little JA, Gallagher G, Perret D, Edwards R \& Dawnay A 1997 Development of enzyme immunoassay for endogenous ouabain-like compound in human plasma. Clinical Chemistry 43 715-722.

Kawamura A, Guo J, Itagaki Y, Bell C, Wang Y, Haupert GT Jr, Magil S, Gallagher RT, Berova N \& Nakanishi K 1999 On the structure of endogenous ouabain. PNAS 96 6654-6659.

Komiyama Y, Kimura Y, Nishimura N, Hara K, Mori T, Okuda K, Munakata M, Masuda M, Murakami T \& Takahashi H 1997 Vasodepressor effects of exercise are accompanied by reduced circulating ouabainlike immunoreactivity and normalization of nitric oxide synthesis. Clinical and Experimental Hypertension 19 363-372.

Laredo J, Hamilton BP \& Hamlyn JM 1994 Ouabain is secreted by bovine adrenocortical cells. Endocrinology 135 794-797.

Lewis LK, Yandle TG, Lewis JG, Richards AM, Pidgeon GB, Kaaja RJ \& Nicholls MG 1994 Ouabain is not detectable in human plasma. Hypertension 24 549-555.

MacPhail S, Thomas TH, Wilkinson R, Davison JM \& Dunlop W 1992 Pregnancy induced hypertension and sodium pump function in erythrocytes. British Journal of Obstetrics and Gynaecology 99 803-807.

Masugi F, Ogihara T, Hasegawa T, Tomii A, Nagano M, Higashimori K, Kumahara K \& Terano Y 1986 Circulating factor with ouabain-like immunoreactivity in patients with primary aldosteronism. Biochemical and Biophysical Research Communications $13541-45$.

Maxwell CV, Quing-Feng T, Seely EW, Repke JT \& Graves SW 1998 Regulation of the sodium pump in pregnancy-related tissues in preeclampsia. American Journal of Obstetrics and Gynecology 179 $28-34$.

Naruse K, Naruse M, Tanabe A, Yoshimoto T, Watanabe Y, Kurimoto F, Horiba N, Tamura M, Inagami T \& Demura H 1994 Does plasma immunoreactive ouabain originate from the adrenal gland? Hypertension 23 (Suppl I) I-102-I-195.

Paci A, Ciarimboli G \& Biver P 1996 Human placenta radioreceptor assay with digoxin and ouabain to detect endogenous digitalis-like factor(s) in human plasma and urine. Clinical Chemistry $\mathbf{4 2}$ 270-278.

Poston L, Morris JF, Wolfe CD \& Hilton PJ 1989 Serum digoxin-like substances in pregnancy-induced hypertension. Clinical Science $\mathbf{7 7}$ 189-194.

Rossi G, Manunta P, Hamlyn JM, Pavan E, De Toni R, Semplicini A \& Pessina AC 1995 Immunoreactive endogenous ouabain in primary aldosteronism and essential hypertension: relationship with plasma renin, aldosterone and blood pressure levels. Journal of Hypertension 13 1181-1191.

Sasaki A, Shinkawa O \& Yoshinaga K 1989 Placental corticotropin-releasing hormone may be a stimulator of maternal adrenocorticotropic hormone secretion in humans. Journal of Clinical Investigation 84 1997-2001.

Schneider R, Wray V, Nimtz M, Lehman WD, Kirch U, Antolovic R \& Schoner W 1998 Bovine adrenals contain, in addition to ouabain, a second inhibitor of the sodium pump. Journal of Biological Chemistry 273 784-792.

Seely EW, Williams GH \& Graves SW 1992 Markers of sodium and volume homeostasis in pregnancy-induced hypertension. Journal of Clinical Endocrinology and Metabolism 74 150-156.

Tymiak AA, Norman JA, Bolgar M, DiDonato G, Lee H, Parker WL, Lo LC, Berova N, Nakanishi K, Haber E \& Haupert GT Jr 1993 Physicochemical characterization of a ouabain isomer isolated from bovine hypothalamus. PNAS 90 8189-8193.

Valdes P Jr \& Graves SW 1985 Protein binding of endogenous digoxin-immunoreactive factors in human serum and its variation with clinical condition. Journal of Clinical Endocrinology and Metabolism 60 1135-1143. 
Worgall S, Hänze J, Wagner R, Peiser C, Lang RE, Sulyok E \& Rascher W 1996 Characterization of ouabain-like immunoreactivity in human urine. Journal of Hypertension 14 623-628.

Yoshimura M, Nishikawa M, Ogasawara H, Horimoto M, Yoshikawa N, Sawaragi I \& Inada M 1993 Measurement of erythrocyte $\mathrm{Na}, \mathrm{K}, \mathrm{ATP}$ ase activity in normal pregnant women. Endocrine Journal 40 171-177.

Zhao N, Lo L-C, Berova N, Nakanishi K, Tymiak AA, Ludens JH \& Haupert GT Jr 1995 Na,K-ATPase inhibitors from bovine hypothalamus and human plasma are different from ouabain: nanogram scale CD structural analysis. Biochemistry 34 9893-9896.

Received 13 August 1999

Revised manuscript received 24 December 1999

Accepted 27 January 2000 